\title{
CONSTRUCTION FUNCTORS FOR TOPOLOGICAL SEMIGROUPS
}

\author{
THOMAS T. BOWMAN
}

\begin{abstract}
In the study of semigroups there often exists a natural decomposition of a semigroup into a semilattice of subsemigroups of known structure. The harder question is, given a semilattice of semigroups to construct from these components a larger semigroup. If in addition, the given semilattice and semigroups are topological, this paper studies the construction of topological semigroups with emphasis on the functorial nature of the construction. It is shown that a semilattice of topological monoids has a unique minimal compatible topology. This is a new characterization of a topology that has been widely used in the special case of compact semigroups, but in the general case it does not necessarily give rise to a Hausdorff topology. It also lacks desirable functorial properties. In \$2, a topological construction is given which satisfies the desired functorial properties. In $\$ 3$, we restrict our attention to subcategories and sufficient conditions for the constructed topologies to be Hausdorff. The constructed topologies of $\S \S 2$ and 3 were motivated by the topologies of dual semigroups in a Pontryagin type duality.
\end{abstract}

The technique of constructing semigroups by combining simpler semigroups or groups has been widely used in algebraic and compact semigroups. A. H. Clifford [6, p. 128] used this method to describe semigroups which are a union of groups and whose set of idempotents forms a semilattice. Also, Hofmann and Mostert [9, pp. 139-142] used this chaining procedure in their description of compact irreducible semigroups. The author in [2] studied construction of compact semigroups over compact Lawson semilattices, and used this method to describe the category of compact Clifford semigroups. A good survey of the construction of compact semigroups is found in [4].

This paper will study the construction of arbitrary topological semigroups. Since it is possible to chain Hausdorff semigroups in a reasonable fashion and not obtain a Hausdorff semigroup, we shall work in the category of not necessarily Hausdorff topological semigroups. A semigroup $S$ will be called a $w$-topological semigroup if $S$ is endowed with a not necessarily Hausdorff topology and the multiplication of $S$ is a continuous map from $S \times S$ into $S$. A $T_{1}$-topological semigroup is a $w$-topological semigroup with a $T_{1}$ topology. We follow general usage in that compactness will also mean Hausdorff. If $Y$ is a partially 
ordered set, then $Y$ will be considered a category by defining $x \rightarrow y$ if and only if $x \geqq y$. An order preserving map of partially ordered sets may also be viewed as a functor. If $X$ is a semilattice and $G$ is a functor from $X$ into the category of semigroups, for $x \geqq y \in X$ denote $G(x \rightarrow y)$ by $G_{y x}$. Let $\mathrm{Cl}(X, G)$ be a disjoint union of $G(x), x \in X . \quad \mathrm{Cl}(X, G)$ becomes a semigroup if multiplication is defined in the following manner: for $s \in G(x), t \in G(y)$ and $u=x \wedge y$, define $s t=G_{u x}(s) G_{u y}(t)$. For $X$ a $T_{1}$-topological semilattice and $G$ a functor from $X$ into the category of $w$-topological semigroups $\mathscr{S}$, this paper studies the topologies on $\mathrm{Cl}(X, G)$ that have the following properties: (i) $\mathrm{Cl}(X, G)$ is a $w$ topological semigroup; (ii) for each $x \in X$, the topology of $G(x)$ coincides with the subspace topology; and (iii) the projection map $p: \mathrm{Cl}(X, G) \rightarrow X$ is continuous. A topology on $\mathrm{Cl}(X, G)$ satisfying (i), (ii) and (iii) is said to be compatible. In this paper, we construct a compatible topology $\mathscr{T}_{1}$ for $\mathrm{Cl}(X, G)$. If $G$ is a functor whose codomain is the category of $w$-topological semigroups with identity, then $\mathscr{T}_{1}$ is the minimal compatible topology. It will be shown in the text why the $\mathscr{T}_{1}$ topology is not completely satisfactory. The major problem is that it lacks certain desirable functorial properties.

A topology $\mathscr{T}_{2}$ will be defined using colimits that will satisfy the functorial properties. By restricting our attention to a subcategory of $T_{1}$-topological semilattices (which contains locally compact $T_{2}$ Lawson semilattices and compact $T_{2}$ semilattices) and by requiring the functors to satisfy certain conditions related to inverse limits, a compatible topology $\mathscr{T}_{0}$ may be defined which avoids the use of colimits. Also, $\mathscr{T}_{0}$ satisfies the desired functorial properties. Sufficient conditions for $\mathscr{T}_{0}$ to be Hausdorff and compact are given.

1. The $\mathscr{T}_{1}$ topology. Let $\mathscr{X}$ denote the category of $T_{1}$ topological semilattices with continuous homomorphisms as morphisms. For $X \in o b(\mathscr{X})$ and $G$ a functor from $\mathscr{X}$ into $\mathscr{S}$, in $\mathrm{Cl}(X, G)$ let $\mathfrak{U}=\left\{p^{-1}(U): U\right.$ open in $\left.X\right\}$. For $k \in X$ and $V \subset G(k)$, define $W(k, V)=\left\{s \in \mathrm{Cl}(X, G): p(s)=u \supsetneqq k\right.$ or $\left.G_{k u}(s) \in V\right\}$. Let $\mathfrak{W}_{1}$ be the collection of all $W(k, V)$ where $k \in X$ and $V$ open in $G(k)$.

THEOREM 1.1. If $X \in o b(\mathscr{X})$ and if $G$ is a functor from $X$ into $\mathscr{P}$, the collection of sets $\mathfrak{U} \cup \mathfrak{W}_{1}$ forms a subbasis for a compatible topology $\mathscr{T}_{1}$ on $\mathrm{Cl}(X, G)$. Furthermore, if $G$ is a functor into the full subcategory of monoids, then $\mathscr{T}_{1}$ is the minimal compatible topology.

Proof. Let $\mathscr{T}_{1}$ be the topology generated by $\mathfrak{U}$ and $\mathfrak{W}_{1}$. We first show that $\mathscr{T}_{1}$ is a semigroup topology. Let $s \in G(x), t \in G(y)$ and $u=x \wedge y$. If $s t \in p^{-1}(U)$ where $U$ is an open set of $X$, there exist open sets $D_{1}$ and $D_{2}$ in $X$ of $x$ and $y$, respectively, such that $D_{1} D_{2} \subset U$. It 
follows that $p^{-1}\left(D_{1}\right)$ and $p^{-1}\left(D_{2}\right)$ are open sets of $s$ and $t$, respectively, in $\mathrm{Cl}(X, G)$ such that $p^{-1}\left(D_{1}\right) p^{-1}\left(D_{2}\right) \subset U$. If $s t \in W(k, V)$, where $W(k, V) \in \mathfrak{B}_{1}$, we consider the cases $u \geq k$ and $u \geqq k$. If $u \geqq k$, then $J_{k}=\{y \in X: y k \neq k\}$ is an open set of $X$, and $s t \in p^{-1}\left(J_{k}\right) \subset$ $W(k, V)$. As proved above there exist $A_{1}, A_{2} \in \mathfrak{U}$ such that $s \in A_{1}$, $t \in A_{2}$ and $s t \in A_{1} A_{2} \subset p^{-1}\left(J_{k}\right) \subset W(k, V)$. If $u \geqq k$, then there exist open neighborhoods $B_{1}$ and $B_{2}$ in $G(k)$ of $G_{u x}(s)$ and $G_{u y}(t)$, respectively, such that $B_{1} B_{2} \subset V$. Now $W\left(k, B_{i}\right)$ and $W\left(k, B_{2}\right)$ are open neighborhoods of $s$ and $t$ respectively, with $W\left(k, B_{1}\right) W\left(k, B_{2}\right) \subset$ $W(k, V)$. It follows that multiplication is a continuous map.

Since for each $U$ open in $X, p^{-1}(U) \in \mathscr{T}_{1}, p$ is a continuous map. Observe that $p^{-1}(U) \cap G(k)$ is either $G(k)$ or void, and also for $W(k, V) \in \mathfrak{B}_{1}$ and $x \in X, W(k, V) \cap G(x)$ is equal to either $G(x)$ or $G_{k x}^{-1}(V)$ and so is open in $G(x)$. Also, $W(k, V) \cap G(k)=$ $V$. Therefore $\mathscr{T}_{1}$ is compatible.

Suppose that $\mathscr{T}$ is a compatible topology and each $G(x), x \in X$, has an identity $l_{x}$. Fix $W(k, V) \in \mathfrak{B}_{1}$ and let $\left\{s_{\alpha}\right\}$ be a net in $\mathrm{Cl}(X, G)-$ $W(k, V)$ which converges to $s$ relative to $\mathscr{T}$. Then $p\left(s_{\alpha}\right) \geqq k$ for all $\alpha$ and hence

$$
\begin{aligned}
G_{k p(s)}(s)= & l_{k} s=l_{k} \lim s_{\alpha}=\lim l_{k} s_{\alpha} \\
= & \lim G_{k p\left(s_{\alpha}\right)}\left(s_{\alpha}\right) \in G(k)-V \text { as } G_{k p\left(s_{\alpha}\right)}\left(s_{\alpha}\right) \in G(k)-V \\
& \text { for all } \alpha .
\end{aligned}
$$

Since $X$ is a $T_{1}$ space with a continuous semilattice multiplication, $p(s) \geqq k$ and so $s \in \mathrm{Cl}(X, G)-W(k, V)$. Hence $W(k, V) \in \mathscr{T}$ and $\mathfrak{B}_{1} \subset \mathscr{T}_{1}$. Clearly by compatibility, $\mathfrak{u} \subset \mathscr{T}$. Hence, $\mathscr{T}_{1} \subset \mathscr{T}$.

Definition 1.2. Let $(\mathscr{X}, \mathscr{S})$ denote the category of all pairs $(X, F)$, where $X \in o b(\mathscr{X})$ and $F$ is a functor from $X$ to $\mathscr{S}$. If $(X, F)$ and $(Y, G)$ are objects of $(\mathscr{X}, \mathscr{S})$, then a morphism from $(X, F)$ to $(Y, G)$ is a pair $(\epsilon, \omega)$, where $\epsilon: X \rightarrow Y$ is a continuous homomorphism and $\omega$ is a natural transformation from $F$ to $G \epsilon$. If $(\epsilon, \omega)$ and $(\delta, \eta)$ are morphisms from $(X, F)$ to $(Y, G)$ and $(Y, G)$ to $(Z, H)$, respectively, composition is defined as follows:

$$
(\delta, \eta) \circ(\epsilon, \omega)=(\delta \circ \epsilon, \eta \epsilon \circ \omega) .
$$

Subcategories of $(\mathscr{X}, \mathscr{S})$ are called semilattice decomposition categories of topological semigroups.

REMARK. In [2], a category similar to the one in the preceding definition was used in the construction of compact semigroups. These 
methods were used further to give a description of the category of compact inverse semigroups whose sets of idempotents are Lawson semilattices: every point has a fundamental system of neighborhoods which are subsemilattices. As in [2], it would be desirable to construct a functor from $(\mathscr{H}, \mathscr{S})$ to $\mathscr{S}$. This will be accomplished by assigning to each object $(X, G)$ of $(\mathscr{H}, \mathscr{S})$ a compatible topology on $\mathrm{Cl}(X, G)$ in such a manner that a functor from $(\mathscr{H}, \mathscr{S})$ to $\mathscr{S}$ is formed in a natural way. It is conjectured that the $\mathscr{T}_{1}$ topology is not functorial.

2. The $\mathscr{T}_{2}$ topology. In this section let $\mathscr{C}$ be a co-complete category of $w$-topological semigroups, and $(\mathscr{X}, \mathscr{C})$ the full subcategory of $(\mathscr{X}, \mathscr{S})$ consisting of the functors whose codomains are $\mathscr{C}$.

REMARK. To stipulate that $\mathscr{C}$ is a co-complete category of $w$ topological semigroups does not imply that $\mathscr{C}$ is a co-complete subcategory of $\mathscr{S}$. For example, the category $\mathscr{S}_{H}$ of Hausdorff topological semigroups is co-complete, but is not a co-complete subcategory of $\mathscr{S}$.

The categories $\mathscr{S}_{H}$ and $\mathscr{S}$ can readily be seen to be complete ${ }^{\dagger}$. The fact that $\mathscr{S}_{H}$ and $\mathscr{S}$ are co-complete follows from the discussion on pages 141-142 of [11], checking the solution set condition by a standard counting argument, and applying Freyd's coadjunct existence theorem.

Definition 2.1. If $X \in o b(\mathscr{X})$ we form a new category called the augmented category of $X$ which will be denoted by $X^{*}$. The objects of $X^{*}$ are the elements of $X$, the open prime ideals of $X$ and the empty set $\varnothing$. If $a, b \in X^{*}$, then $a \rightarrow b$ if and only if one of the following holds: (i) $a, b \in X$ and $a \geqq b$, (ii) $a \in X, b$ is an open prime ideal and $a \notin b$, (iii) $a$ and $b$ are open prime ideals of $X$ and $a \supseteq b$, or (iv) $b=\varnothing$.

If $(X, G) \in o b(\mathscr{X}, \mathscr{C})$, we extend the functor $G$ to a functor from $X^{*}$ to $\mathscr{C}$, which we also denote by $G$, by defining for an open prime ideal $a$ of $X$ (or for $a=\varnothing)$,

$$
G(a)=\operatorname{colim}\left\{G(x) ; G_{y x}: y \leqq x \notin a\right\} .
$$

For $x \in X$ and $x \notin a, G_{a x}$ is the canonical map defined by the colimit diagram. For open prime ideals $a \geqq b$ (or $b=\varnothing$ ), $G_{b a}: G(a) \rightarrow G(b)$ is defined by the uniqueness of the universal property of colimits.

Suppose $(X, G)$ is an object of $(\mathscr{X}, \mathscr{C})$. For each open prime ideal $a$ (or $a=\varnothing)$ and $U \subseteq G(a)$, let $W(a, U)=\{s \in C l(X, G): u=p(s) \in a$ or $\left.G_{a u}(s) \in U\right\}$. Let $\mathfrak{W}_{2}$ be the collection of all sets of the form $W(a, U)$, where $a$ is an open prime ideal of $X$ or $a=\varnothing$ and $U$ is open in $G(a)$.

THEOREM 2.2. For $(X, G)$ an object of $(\mathscr{X}, \mathscr{C})$, the collection of sets

\footnotetext{
For the completeness of $\mathscr{S}_{H}$ and $\mathscr{S}$ we must allow the void semigroup.
} 
$\mathfrak{H} \cup \mathfrak{B}_{2}$ forms a subbasis for a compatible semigroup topology $\mathscr{T}_{2}$ on $\mathrm{Cl}(X, G)$.

Proof. Let $\mathscr{T}_{2}$ be the topology generated by $\mathfrak{U} \cup \mathfrak{B}_{2}$. We first show that $\mathscr{T}_{2}$ is a semigroup topology for $\mathrm{Cl}(X, G)$. For an element $x \in X$, since $X$ is $T_{1}$, the set $J_{x}=\{y \in X: y x \neq x\}$ is an open prime ideal. Also since $x$ is the least element of $X-J_{x}, G(x)=$ $\operatorname{colim}\left\{G(z), G_{y z}: y \leqq z \notin J_{x}\right\}$. For an open set $U$ of $G(x)$, we have $W(x, U)=W\left(J_{x}, U\right)$ which implies that $\mathfrak{B}_{1} \subset \mathfrak{B}_{2}$. In view of the proof of 1.1 it suffices to consider the case $s t \in W(a, V)$ where $a$ is an open prime ideal or $a=\varnothing$. If $p(s) p(t)=p(s t) \in a$ then let $U_{1}$ and $U_{2}$ be open sets containing $p(s)$ and $p(t)$, respectively, such that $U_{1} U_{2} \subset a$. It follows that $s \in p^{-1}\left(U_{1}\right), \quad t \in p^{-1}\left(U_{2}\right)$, and $p^{-1}\left(U_{1}\right) p^{-1}\left(U_{2}\right) \subset p^{-1}(a) \subset$ $W(a, V)$. If $p(s t) \notin a$ then $p(s) \notin a$ and $p(t) \notin a$. Thus $G_{a p(s)}(s) G_{a p(t)}(t) \in V$. Using continuity of multiplication on $G(a)$ one obtains open sets $V_{1}$ and $V_{2}$ containing $G_{a p(s)}(s)$ and $G_{a p(t)}(t)$, respectively, such that $V_{1} V_{2} \subset V$. It follows that $s \in W\left(a, V_{1}\right), t \in W\left(a, V_{2}\right)$ and $W\left(a, V_{1}\right) W\left(a, V_{2}\right) \subset W(a, V)$.

Since $\mathscr{T}_{1} \subset \mathscr{T}_{2}$, to show that $G(x)$ inherits its original topology, it suffices to observe that if $W(a, V) \in \mathfrak{W}_{2}$ then $W(a, V) \cap G(x)$ is either $G(x)$ or $G_{a x}^{-1}(V)$.

Continuity of $p$ is obvious, as $\mathscr{T}_{1} \subset \mathscr{T}_{2}$.

THEOREM 2.3. If $(X, F)$ is an object of $(\mathscr{X}, \mathscr{C})$, define $\mathrm{Cl}_{2}(X, F)$ to be $\mathrm{Cl}(X, F)$ with the $\mathscr{T}_{2}$ topology, and if $(\epsilon, \omega)$ is a morphism from $(X, F)$ to $(Y, G)$ define $\mathrm{Cl}_{2}(\epsilon, \omega): \mathrm{Cl}_{2}(X, F) \rightarrow \mathrm{Cl}_{2}(Y, G)$ by

$$
\mathrm{Cl}_{2}(\epsilon, \omega)(s)=\omega_{x}(s) \text { if } s \in F(x) .
$$

Then $\mathrm{Cl}_{2}$ is a functor from $(\mathscr{Q}, \mathscr{C})$ to $\mathscr{S}$.

Proof. It will be shown that $\mathrm{Cl}_{2}(\epsilon, \omega)$ is continuous. That $\mathrm{Cl}_{2}(\epsilon, \omega)$ is a homomorphism and that $\mathrm{Cl}_{2}$ preserves composition of morphisms are straightforward verifications. Let $f=\mathrm{Cl}_{2}(\epsilon, \omega)$. For $U$ an open set of $Y, f^{-1}\left(p^{-1}(U)\right)=p^{-1}\left(\epsilon^{-1}(U)\right)$ which is an open set of $\mathrm{Cl}_{2}(X, F)$. For $W(a, V)$ a subbasic open set of $\mathrm{Cl}_{2}(Y, G), b=\epsilon^{-1}(a)$ is an open prime ideal of $X$ or $b=\varnothing$. For $x \geqq y \in X-b, G_{a \in(x)}{ }^{\circ} \omega_{x}=G_{a \in(y)}{ }^{\circ} \omega_{y} \circ F_{y x}$. Since $F(b)=\operatorname{colim}\left\{F(x), F_{y x}: y \leqq x \in X-b\right\}$, there exists a unique continuous homomorphism $\omega_{b}: F(b) \rightarrow G(a)$ such that for all $x \in X-b$, $\omega_{b} \circ F_{b x}=G_{a \in(x)} \circ \omega_{x}$. It follows that $V^{\prime}=\omega_{b}^{-1}(V)$ is an open set of $F(b)$. It will be shown that $W\left(b, V^{\prime}\right)=f^{-1}(W(a, V))$. Suppose $s \in W\left(b, V^{\prime}\right)$. If $p(s) \in b$, then $p(f(s))=\epsilon(p(s)) \in a$. By definition of $W(a, V), f(s) \in W(a, V)$. If $u=p(s) \in X-b$, then 


$$
G_{a \in(u)} \circ f(s)=G_{a \in(u)} \circ \omega_{x}(s)=\omega_{b} \circ F_{b u}(s) \in \omega_{b}\left(V^{\prime}\right) \subseteq V
$$

Hence $f(s) \in W(a, V)$. It follows that $W\left(b, V^{\prime}\right) \subseteq f^{-1}(W(a, V))$. Suppose $t \in f^{-1}(W(a, V))$. If $p(t) \in b$, then $t \in W\left(b, V^{\prime}\right)$. If $v=$ $p(t) \in X-b$, then $G_{a \in(v)} \circ \omega_{v}(t)=\omega_{b} \circ F_{b v}(t) \in V$. Thus $F_{b v}(t) \in \omega_{b}^{-1}(V)=$ $V^{\prime}$ and $t \in W\left(b, V^{\prime}\right)$. It follows that $W\left(b, V^{\prime}\right)=f^{-1}(W(a, V))$.

The inverse images of subbasic open sets of $\mathrm{Cl}_{2}(Y, G)$ are open in $\mathrm{Cl}_{2}(X, F)$, which implies that the map $f=\mathrm{Cl}_{2}(\epsilon, \omega)$ is continuous.

THEOREM 2.4. Let $G$ be a functor from a Hausdorff semilattice $X$ to a co-complete category $\mathscr{C}_{H}$ of Hausdorff topological semigroups. For each $x \in X$, define the map $\varphi_{x}: G(x) \rightarrow \Pi G(a)$ (a an open prime ideal or $a=\varnothing)$ with $x \in(X-a)^{\circ}$ by $\varphi_{x}(s)=\left\langle G_{a x}(s)\right\rangle$. If for each $x \in X$, the map $\varphi_{x}$ is one-to-one, then $\mathscr{T}_{2}$ is Hausdorff.

Proof. Suppose $s, t \in \mathrm{Cl}_{2}(X, G)$. If $p(s) \neq p(t)$, then $s$ and $t$ may be separated by inverse images of open sets of $X$. Suppose $u=p(s)=$ $p(t)$. Since the map $\varphi_{u}$ is one-to-one, there exists an open prime ideal $a$ of $X$ such that $u \in(X-a)^{\circ}$ and $G_{a u}(s) \neq G_{a u}(t)$. Since $G(a)$ is Hausdorff, there exist disjoint open neighborhoods $V_{1}$ of $s$ and $V_{2}$ of $t$. Then $W\left(a, V_{1}\right) \cap p^{-1}\left((X-a)^{\circ}\right)$ and $W\left(a, V_{2}\right) \cap p^{-1}\left((X-a)^{\circ}\right)$ are disjoint open neighborhoods of $s$ and $t$, respectively.

REMARK. Any inverse limit preserving functor from a compact Lawson semilattice to compact semigroups satisfies the conditions of 2.4. It seems plausible that a modification of 2.4 may generalize the chaining results of [2] to a larger class of compact semilattices.

3. The $\mathscr{T}_{0}$ topology. For $X \in \mathscr{X}$ and $x \in X$, let $\mathscr{L}(x)=$ $\left\{y \in X: x \in U(y)^{\circ}\right\}$ where $U(y)=\{z \in X: z \geqq y\}$.

Definition 3.1. A semilattice $X$ of $\mathscr{X}$ is said to be neighborhood prime bounded (NPB) if for each open prime ideal $R$ (or $R=\varnothing$ ) and every $x \in(X-R)^{\circ}, \mathscr{L}(x) \cap(X-R) \neq \varnothing$.

REMARK. Any compact semilattice or any locally compact Lawson semilattice is NPB. Also, note that for a NPB semilattice $\mathscr{L}(x)$ is a (upwards) directed subset.

The next proposition shows that when the semilattice is NPB the conditions of 2.4 may be simplified.

Proposition 3.2. Suppose $X$ is a NPB semilattice of $\mathscr{X}$ and $G$ is a functor from $X$ to a co-complete category $\mathscr{C}_{H}$ of Hausdorff semigroups. For each $x \in X$, define the map $\eta_{x}: G(x) \rightarrow \Pi G(z), z \in$ 
$\mathscr{L}(x)$, by $\eta_{x}(s)=\left\langle G_{z x}(s)\right\rangle$. Then for $x \in X, \eta_{x}$ is one-to-one if and only if $\varphi_{x}$ (of 2.4) is one-to-one.

Proof. Suppose $\eta_{x}$ is one-to-one. For $s \neq t$ elements of $G(x)$, there exists a $y \in \mathscr{L}(x)$ such that $G_{y x}(s) \neq G_{y x}(t)$. Now $b=$ $\{z \in X: z y \neq y\}$ is an open prime ideal, and since $y$ is the least element of $X-b, G(b)=G(y)$ and $G_{b x}=G_{y x}$. Therefore, $G_{b x}(s) \neq G_{b x}(t)$ which implies $\varphi_{x}(s) \neq \varphi_{x}(t)$.

Suppose $\varphi_{x}$ is one-to-one. There exists an open prime ideal $a$ (or $a=\varnothing)$ such that $x \in(X-a)^{\circ}$ and $G_{a x}(s) \neq G_{a x}(t)$. Since $X$ is NPB there exists an element $z \in \mathscr{L}(x) \cap(X-a)$. Since $G_{a x}=G_{a z} \circ G_{z x}$, $G_{a x}(s) \neq G_{a x}(t)$ implies $G_{z x}(s) \neq G_{z x}(t)$ which implies $\eta_{x}(s) \neq \eta_{x}(t)$.

Definition 3.3. If $X$ is a NPB semilattice of $\mathscr{X}$, a functor is said to be (semi-) continuous from below if for each $x \in X, \eta_{x}$ is an (embedding) iseomorphism (into) onto inv $\lim \left(G(z), G_{y x}: y \leqq z \in \mathscr{L}(x)\right\} \subset \Pi G(z)$, $z \in \mathscr{L}(x)$.

Notation. Let $\mathscr{Y}$ denote the full subcategory in $\mathscr{X}$ of all NPB semilattices. Also, let $[\operatorname{scb}(\mathscr{Y}, \mathscr{S})] c b(\mathscr{Y}, \mathscr{S})$ denote the full subcategory in $(\mathscr{X}, \mathscr{Y})$ of all pairs $(X, G)$, where $X \in o b(\mathscr{Y})$ and $G$ is [semi-] continuous from below.

Suppose $(X, G)$ is an object of $\operatorname{scb}(\mathscr{Y}, \mathscr{Y})$. For $k \in X$ and $A$ a subset of $G(k)$, define $V(k, A)=\left\{s \in \mathrm{Cl}(X, G): p(s)=u \in U(k)^{\circ}\right.$ and $\left.G_{k u}(s) \in A\right\}$. Let $\mathcal{V}$ be the collection of all $V(k, A)$, where $k \in X$ and $A$ is open in $G(k)$.

THEOREM 3.4. If $(X, G)$ is an object of $\operatorname{scb}(\mathscr{Y}, \mathscr{S})$, then the collection of all $A \cap B$, where $A \in \mathscr{V}$ and $B \in \mathfrak{U}$, forms a basis for a compatible topology $\mathscr{T}_{0}$ for $\mathrm{Cl}(X, G)$.

Proof. Let $\mathscr{T}_{0}$ be the topology generated by the collection of sets $\mathscr{V} \cup \mathfrak{H}$. We first show that the collection of all sets $A \cap B$, where $A \in \mathcal{V}$ and $B \in \mathfrak{H}$, forms a basis for the topology $\mathscr{T}_{0}$. If $s \in \mathrm{Cl}(X, G)$, then a fundamental basic neighborhood $D$ of $s$ is of the form

$$
D=p^{-1}\left(U_{1}\right) \cap \cdots \cap p^{-1}\left(U_{r}\right) \cap V\left(k_{1}, W_{1}\right) \cap \cdots \cap V\left(k_{n}, W_{n}\right)
$$

where $U_{1}, \cdots, U_{r}$ are open in $X, k_{1}, \cdots, k_{n} \in X$ and $W_{i}$ is open in $G\left(k_{i}\right)$. Let $U=U_{1} \cap \cdots \cap U_{r}$. For each $i \in\{1, \cdots, n\}, \quad J_{k_{i}}=$ $\left\{x \in X: x k_{i} \neq k_{l}\right\}$ is an open prime ideal of $X$, and $J=\cup J_{k_{i}}(i=1, \cdots, n)$ is an open prime ideal of $X$ such that $x \in(X-J)^{\circ}$. Since $X$ is NPB, there exists a $k \in X$ such that $x \in U(k)^{\circ}$ and $k \in X-J$. For each $i \in\{1, \cdots, n\}, G_{k_{i k}}{ }^{\circ} G_{k p(s)}(s)=G_{k i p(s)}(s)$ which implies $G_{k, k}^{-1}\left(W_{i}\right)$ is an open 
neighborhood of $G_{k p(s)}(s)$ in $G(k)$. Let $W=\cap G_{k, k}^{-1}\left(W_{i}\right)(i=1, \cdots, n)$. Then $s \in V(z, W) \subset V\left(k_{1}, W_{1}\right) \cap \cdots \cap V\left(k_{n}, W_{n}\right)$. Thus $s \in p^{-1}(U) \cap$ $V(z, W) \subset D$. Observe that $\mathfrak{U}$ is contained in the basis because for any $x \in X, W(x, G(x))=\mathrm{Cl}(X, G)$, and $\mathscr{V}$ is contained in the basis since $p^{-1}(X)=\mathrm{Cl}(X, G)$.

The proof that $\mathscr{T}_{0}$ is a semigroup topology is similar to that found in the proofs of 1.1 and 2.2 .

We now show that $\mathscr{T}_{0}$ is a compatible topology. The continuity of $p$ follows immediately from the fact that $\mathfrak{U} \subset \mathscr{T}_{0}$. It is clear that for each $k \in X$ the relative subspace topology of $G(k)$ with respect to $\mathscr{T}_{0}$ is weaker than the topology of $G(k)$. Denote inv $\lim \left\{G(y), G_{z y}: z \leqq y \in\right.$ $\mathscr{L}(k)\}$ by $H(k)$. It is given that the map $\eta_{k}: G(k) \rightarrow H(k) \subset \Pi G(y)$, $y \in \mathscr{L}(k)$, is an embedding. It follows that the $\eta_{k}$ inverse images of a subbasis of $H(k)$ form a subbasis of $G(k)$. If for $u \in \mathscr{L}(k)$, $\pi_{u k}: H(k) \rightarrow G(u)$ denotes the projection, then $G_{u k}=\pi_{u k} \circ \eta_{k}$. The collection of all sets $\pi_{u k}^{-1}\left(B_{u}\right), u \in \mathscr{L}(k)$ and $B_{u}$ an open subset of $G(u)$, forms a subbasis for the topology of $H(k)$. But $\eta_{k}^{-1} \pi_{u k}^{-1}\left(B_{u}\right)=$ $V\left(u, B_{u}\right) \cap G(k)$ which implies the subspace topology contains a subbasis for the topology of $G(k)$. It follows that the topology of $G(k)$ is the same as the subspace topology induced from $\mathscr{T}_{0}$. 2.3 .

In the following theorem, $\mathrm{Cl}_{0}$ is defined in the same fashion as $\mathrm{Cl}_{2}$ in

THEOREM 3.5.

(i) $\mathrm{Cl}_{0}$ is a functor from $\operatorname{scb}(\mathscr{Y}, \mathscr{S})$ to $\mathscr{S}$.

(ii) If $\mathscr{Y}_{H}$ is the full subcategory of Hausdorff NPB semilattices, $\mathrm{Cl}_{0}$ restricted to the subcategory $\operatorname{scb}\left(\mathscr{Y}_{H}, \mathscr{S}_{H}\right)$ is a functor into $\mathscr{S}_{H}$.

(iii) If $\mathscr{X}_{C}$ is the full subcategory of compact semilattices and $\mathscr{S}_{C}$ is the full subcategory of compact semigroups, then $\mathrm{Cl}_{0}$ restricted to $c b\left(\mathscr{X}_{C}, \mathscr{S}_{C}\right)$ is a functor into $\mathscr{S}_{C}$.

Proof of (i). What needs to be shown is that if $(\epsilon, \omega):(X, F) \rightarrow(Y, G)$ is a morphism in $\operatorname{scb}(\mathscr{Y}, \mathscr{Y})$, then $f=\mathrm{Cl}_{0}(\epsilon, \omega)$ from $\mathrm{Cl}_{0}(X, F)$ to $\mathrm{Cl}_{0}(Y, G)$ is continuous. Let $s \in \mathrm{Cl}_{0}(X, F)$ and let $p^{-1}(U) \cap V(k, W)$ be a fundamental open neighborhood of $f(s)$ as described in 3.4. Now $f^{-1}\left(p^{-1}(U)\right)=p^{-1}\left(\epsilon^{-1}(U)\right)$ which is open in $\mathrm{Cl}_{0}(X, F)$. If $J_{k}=\{y \in Y: y k \neq k\}$, then $P=\epsilon^{-1}\left(J_{k}\right)$ is an open prime ideal of $X$ such that $p(s) \in(X-P)^{\circ}$. Since $X$ is NPB, there exists a $z \in \mathscr{L}(x)$ such that $z \in X-P$. If $W^{\prime}=\omega_{z}^{-1}\left(G_{k \in(z)}^{-1}(W)\right)$, then $W^{\prime}$ is an open subset of $F(z)$. Now $p^{-1}\left(\epsilon^{-1}(U)\right) \cap V\left(z, W^{\prime}\right)$ is an open neighborhood of $s$ which is contained in $f^{-1}\left(p^{-1}(U) \cap V(k, W)\right)$ which implies $f$ is continuous at $s$.

Proof of (ii). Suppose $(X, G)$ is an object of $\operatorname{scb}\left(\mathscr{Y}_{H}, \mathscr{Y}_{H}\right)$ and 
further suppose $s \neq t$ are elements of $\mathrm{Cl}_{0}(X, G)$. If $p(s) \neq p(t)$, then in $X$ there exist disjoint open neighborhoods $U_{1}$ of $p(s)$ and $U_{2}$ of $p(t)$. Then $p^{-1}\left(U_{1}\right)$ and $p^{-1}\left(U_{2}\right)$ are disjoint open neighborhoods of $s$ and $t$ respectively. Now assume $p(s)=p(t)$. Since $\eta_{x}$ is one-to-one, there exists an element $z \in \mathscr{L}(x)$ such that $G_{z x}(s) \neq G_{z x}(t)$. In $G(z)$, there exist disjoint open neighborhoods $W_{1}$ of $G_{z x}(s)$ and $W_{2}$ of $G_{z x}(t)$. It follows that $V\left(z, W_{1}\right)$ and $V\left(z, W_{2}\right)$ are disjoint open neighborhoods of $s$ and $t$ respectively.

Proof of (iii). Let $(X, G)$ be an object of $c b\left(\mathscr{L}_{C}, \mathscr{S}_{C}\right)$. Suppose $\left\{s_{\alpha}\right\}$, $\alpha \in \Lambda$, is a net in $\mathrm{Cl}_{0}(X, G)$. Letting $x_{\alpha}=p\left(s_{\alpha}\right)$, a net in $X$ is obtained. Since $X$ is compact, the net $\left\{x_{\alpha}\right\}$ has a convergent subnet, so it may be assumed without loss of generality that $\left\{x_{\alpha}\right\}$ converges to $x$. Let $\left\langle d_{z}\right\rangle \in \Pi G(z), z \in \mathscr{L}(x)$. Define the net $\left\{t_{\alpha}\right\}, \alpha \in \Lambda$, in $\Pi G(z), z \in$ $\mathscr{L}(x)$, by

$$
t_{\alpha}(z)=\left\{\begin{array}{l}
d_{z}, x_{\alpha} \supsetneqq z \\
G_{z x_{\alpha}}\left(s_{\alpha}\right), x_{\alpha} \geqq z .
\end{array}\right.
$$

Since $\Pi G(z), z \in \mathscr{L}(x)$, is compact, it may be assumed that $\left\{t_{\alpha}\right\}, \alpha \in \Lambda$, converges to $t \in \Pi G(z), z \in \mathscr{L}(x)$. For $i \leqq j \in \mathscr{L}(x)$ define

$$
P_{i j}=\left\{c \in \Pi G(z), z \in \mathscr{L}(x): G_{i j}(c(j))=c(i)\right\} .
$$

Now inv $\lim \left\{G(z), G_{y z}: y \leqq z \in \mathscr{L}(x)\right\}=\cap P_{i j}(i \leqq j \in \mathscr{L}(x))$. If $k \geqq$ $l \in \mathscr{L}(x)$, since $\lim x_{\alpha}=x$, there exists an $\alpha_{0} \in \Lambda$ such that for $\alpha \geqq \alpha_{0}$, $x_{\alpha} \in U(k)^{0}$ and $G_{l k}\left(t_{\alpha}(k)\right)=t_{\alpha}(l)$. Thus for $\alpha \geqq \alpha_{0}, t_{\alpha} \in P_{l k}$ and since $P_{l k}$ is closed, $\quad t=\lim t_{\alpha} \in P_{l k}$. Hence $\quad t \in \cap P_{i j} \quad(i \leqq j \in \mathscr{L}(x))=$ inv $\lim \left\{G(z), G_{y z}: y \leqq z \in \mathscr{L}(x)\right\}$. Since $\eta_{x}$ is an iseomorphism let $s$ be the element of $G(x)$ such that $\eta_{x}(s)=t$, i.e., for $k \in \mathscr{L}(x), G_{k x}(s)=$ $t(k)$. To establish that $\lim s_{\alpha}=s$, it suffices to show that for any $k \in \mathscr{L}(x)$ and any open subset $W$ of $G_{k x}(s)$ in $G(k)$ that $\left\{s_{\alpha}\right\}$ is finally in $V(k, W)$. Now $\lim x_{\alpha}=x$ implies there is a $\beta_{0} \in \Lambda$ such that for $v \geqq \beta_{0}$, $x_{v} \in U(k)$. Restricted to $v \geqq \beta_{0}$,

$$
\lim G_{k x_{v}}\left(s_{v}\right)=\lim t_{v}(k)=t(k)=G_{k x}(s) \in W .
$$

Thus $\left\{s_{\alpha}\right\}$ is finally in $V(k, W)$ which implies $\left\{s_{\alpha}\right\}$ converges to $s$ in $\mathrm{Cl}_{0}(X, G)$.

REMARK. If $(X, G)$ is an object of $\operatorname{scb}(\mathscr{Y}, \mathscr{S})$, then on $\operatorname{Cl}(X, G)$, $\mathscr{T}_{0} \subset \mathscr{T}_{1} \subset \mathscr{T}_{2}$. Suppose $\mathcal{M}$ is the full subcategory in $\mathscr{S}$ of topological monoids. If $(X, G)$ is an object of $\operatorname{scb}(\mathscr{Y}, \mathcal{M})$, then by $1.1, \mathscr{T}_{0}=\mathscr{T}_{1}$ on 
$\mathrm{Cl}(X, G)$. So $\mathrm{Cl}_{1}$ may be viewed as a functor on $\operatorname{scb}(\mathscr{Y}, \mathcal{M})$. It is in this category that construction methods have been applied. The topologies used in [2], [5], [7], [8], [9] and [10] are the $\mathscr{T}_{0}=\mathscr{T}_{1}$ topology. In the duality Theorem 3.4 of [3], condition $B$ is similar to continuity from below. By virtue of 1.1, the unique topology on a semigroup that is naturally isomorphic to its second dual is the $\mathscr{T}_{0}$ topology. If the semilattice is locally compact, 2.4 of [1] implies the generating idempotents in $\mathscr{L}(x)$ form a cofinal subset of $\mathscr{L}(x)$. Therefore, condition $B$ is equivalent to continuity from below, and $\mathscr{T}_{0}=\mathscr{T}_{1}$. A small amount of study will reveal that the topology (2.2 of [2]) of the character semigroup is a modification of the $\mathscr{T}_{2}$ topology.

\section{REFERENCES}

1. J. W. Baker and N. J. Rothman, Separating points by semicharacters in topological semigroups, Proc. Amer. Math. Soc., 21 (1969), 235-239.

2. T. T. Bowman, A construction principle and compact Clifford semigroups, Semigroup Forum, 2 (1971), 343-353.

3. - Analogue of Pontryagin character theory for topological semigroups, Proc. Amer. Math. Soc., 46 (1974), 97-105.

4. J. H. Carruth, Building $S$ from $S / \mathscr{K}$, Proceedings of the Second Florida Symposium on Automata and Semigroups, University of Florida (1971).

5. J. H. Carruth and J. D. Lawson, Semigroups through semilattices, Trans. Amer. Math. Soc., 152 (1970), 597-608.

6. A. H. Clifford and G. B. Preston, The Algebraic Theory of Semigroups, Vol. I, Mathematical Surveys, 7, Amer. Math. Soc., 1961.

7. R. O. Fulp, Character semigroups of locally compact inverse semigroups, Proc. Amer. Math. Soc., 1 (1969), 719-724.

8. T. E. Hays, Monotonicity, ML-semigroups and $\leqq(\mathscr{Y})$-stability, dissertation, University of Tennessee, 1971.

9. K. H. Hofmann and P. S. Mostert, Elements of Compact Semigroups, Charles E. Merrill Books, Inc., Columbus, Ohio, 1966.

10. M. W. Mislove, Semigroups over trees, submitted, Trans. Amer. Math. Soc.

11. B. Mitchell, Theory of Categories, Academic Press, 1965.

Received May 29, 1973 and in revised form July 25, 1974.

UNIVERSITY OF FLORIDA

GAINESVILLE, FLORIDA 32611 